\title{
Management of the deaf child
}

\section{A D K S N Yasawardene ${ }^{1}$}

Sri Lanka Journal of Child Health, 2003; 32: 71-4

(Key words: deafness, child)

Hearing impairment is the commonest sensory disorder worldwide $^{1}$. Deafness in a child may be congenital or acquired. Congenital deafness is mostly sensorineural and has an incidence of 1-1.5 per 1000 children $^{2}$. Acquired deafness is mostly conductive and is commonly due to glue ear or otitis media with effusion (OME). Up to $30 \%$ of preschool children can have OME especially in cold climates ${ }^{2}$.

\section{Causes of hearing impairment in childhood}

Relative contribution of genetic and environmental causes to hearing impairment is determined by factors such as consanguinity, infection control, immunization and provision of antenatal/perinatal care in a given society ${ }^{1}$. In the developed world approximately $50 \%$ of childhood deafness is caused by genetic factors. About $30 \%$ of genetic deafness is said to be syndromic i.e. deafness is part of a known syndrome. $75-80 \%$ with non-syndromic genetic hearing loss have an autosomal recessive cause. $10-15 \%$ have an autosomal dominant aetiology and the rest are caused by X-linked, mitochondrial or chromosomal causes ${ }^{1}$.

Non-genetic causes of early-onset deafness are listed in table 1. Post-meningitic deafness is mainly caused by Streptococcus pneumoniae and antibiotic treatment delayed for even 24 hours is a well recognized risk factor for developing hearing loss ${ }^{3}$.

\section{Table 1}

Non-genetic causes of early-onset deafness

Maternal Diabetes

Toxaemia of pregnancy

Ototoxic drugs

Teratogenic chemicals

Infections - Rubella, cytomegalovirus, herpes simplex, toxoplasma, syphilis, parvovirus, human immunodeficiency virus, chickenpox, mumps, meningitis, encephalitis, septicaemia

Asphyxia neonatorum

Prematurity and small for gestational age

Neonatal hyperbilirubinaemia

Delayed auditory maturation

${ }^{1}$ Consultant Ear, Nose and Throat Surgeon, Lady Ridgeway Hospital, Colombo.

\section{Diagnosis}

Although diagnosis is best achieved as early as possible in congenital deafness, it is not always easy due to following factors:

- $\quad 30-40 \%$ of deafness occurs as an isolated disorder.

- Small babies cannot complain of deafness and up to $30 \%$ parents/caretakers are unaware of problem ${ }^{2}$.

- Instead of a single test, many tests, according to the child's age, are needed and may have to be repeated to arrive at a firm diagnosis of deafness.

- All these tests need special equipment and specially trained personnel.

It is recommended that diagnosis be made and rehabilitation commenced before six months of age to achieve the best results. A consultant-led multidisciplinary team, including otolaryngology, audiology, paediatrics, genetics, plastic surgery, deaf education. speech therapy, psychology and social services, best achieves this goal. This multidisciplinary approach is best delivered in a hospital-based set up, as these children may have other special health needs ${ }^{2}$.

\section{Tests of hearing}

Tests of hearing can be objective (child's cooperation not needed) or behavioural (depends on child's ability and cooperation) (Table 2).

Table 2

Tests of hearing

\section{Objective Tests}

- Oto-Acoustic Emissions (OAE)

- Brainstem Evoked Response Audiometry (BSER) or Auditory Brainstem Response (ABR)

- Auditory response cradle

- Impedance audiometry 


\section{Behavioural Tests}

- Distraction tests

- Visual Reinforcement Audiometry (VRA)

- Play Audiometry

- $\quad$ Pure tone Audiometry (PTA)

- Speech Audiometry

\section{Screening for deafness}

Since there is a possibility of a delay in diagnosis of deafness until child shows language problems, screening for deafness was proposed to increase early detection. Screening can be selective for those with identifiable risk factors (table 3) or universal (for all children).

Table 3

\section{At risk factors}

Family history of deafness

Consanguinity

Maternal infections

Use of ototoxic drugs

Congenital defects

Prematurity \& small for gestational age

Asphyxia neonatorum

Neonatal hyperbilirubinaemia

Neonatal infections - meningitis, septicaemia

48 hour stay in neonatal intensive care unit (NICU)

Head injury

Syndromes with deafness

Selective screening will not pick up $30-40 \%$ of hearing impaired as they have hearing loss as an isolated defect. In the United States of America, up to $50 \%$ children could not be identified early enough by selective screening and universal screening was advocated ${ }^{4}$. Universal screening should be done in the neonatal period, at 8 months, at school entry and in any child after head injury or meningitis, with appropriate tests (table 4). All failures are referred to a paediatric audiology-testing centre.
Table 4

Universal screening stages

Neonatal period - OAE (NICU Babies - Automated ABR \& OAE)

8 months - Distraction test or Automated VRA

School entry - Audiometric Sweep

Any child - After head injury or meningitis

\section{Paediatric audiometry}

Accurate hearing assessment in children is best achieved in a child-friendly environment by testers, well experienced in techniques of paediatric audiometry. The testing room area should be at least 16 square metres with ambient noise level less than $30 \mathrm{~dB}$. It is necessary to select tests appropriate to child's age and development, also considering other handicaps like visual defects. Tests that can be used at different age groups are listed in table 5 .

Table 5

\section{Hearing tests for various age groups}

Under Six months - OAE, BSER and Auditory response cradle with high frequency $(660 \mathrm{Htz})$ impedance testing

7-17 months - Distraction tests

7-30 months - VRA

2-5 years - Play audiometry

$41 / 2$ years onwards - PTA

2 years onwards - Speech audiometry

A battery of tests, rather than a single test, is used for definitive diagnosis of deafness. Necessary components of the final diagnosis and the appropriate tests are listed in table 6 .

Table 6

Components of final diagnosis of deafness and appropriate tests

\begin{tabular}{|l|l|}
\hline \multicolumn{1}{|c|}{ Components of diagnosis } & \multicolumn{1}{c|}{ Tests } \\
\hline 1. Degree of Hearing Impairment & Air Conduction ABR thresholds measured to Click Stimuli \\
\hline 2. Type of Hearing Impairment & Bone Conduction ABR thresholds \\
& $\begin{array}{l}\text { Plotting of the Latency Intensity Function from } \\
\text { Air and Bone conduction ABR }\end{array}$ \\
Tympanometry \\
\hline 3. Configuration of Hearing Impairment & Tone Pip ABR \\
\hline 4. Behavioural Confirmation of Hearing Impairment & Behavioural Observation Audiometry Visual \\
& Reinforcement Audiometry Infant Distraction Test \\
\hline
\end{tabular}




\section{Non-audiological evaluation of the deaf child}

Non-audiological evaluation is essential to find a possible aetiological diagnosis and to identify other factors affecting rehabilitation. Detailed gestational, perinatal, family, ear nose and throat (ENT), other medical, developmental, language and speech, social and educational history must be obtained. Examination should include ENT system, eyes, development and dysmorphic features. Relevant investigations are listed in table 7. Those with persistent glue ear (OME) should be investigated for submucous cleft palate, mucociliary abnormalities, cranio-facial defects and adenoidal hypertrophy.

\section{Table 7 Investigations}

Ophthalmologic assessment

Renal ultrasound

Urinalysis - casts, red blood cells, mucopolysaccharides

Viral studies - infants $<6$ months of age

High resolution CT scan of temporal bone

Speech and development assessment

ECG for Jervell Lange-Nielson syndrome

Thyroid function

Full blood count \& ESR for Cogan Syndrome

Autoimmune profile

Vestibular (balance) assessment

Family Screening

\section{Genetic diagnosis of deafness}

Hereditary deafness has proved to be extremely heterogeneous genetically with more than 40 genes mapped or cloned for non-syndromic dominant deafness and 30 for non-syndromic autosomal recessive deafness. In spite of significant advances in understanding of the molecular basis of hearing loss, identifying precise genetic cause in an individual remains difficult. Consequently, it is important to exclude syndromic causes of deafness by clinical and special investigation and to use all available phenotypic clues for diagnosis. Molecular screening of GJB2 (Connexin 26) gene can be undertaken in all cases of non-syndromic deafness where cause cannot be identified, since it is a common cause of recessive hearing impairment, the screening is straightforward, and the phenotype unremarkable. Mitochondrial inheritance of hearing loss should be considered in all multigeneration families, particularly if there is a history of exposure to aminoglycoside antibiotics, since genetic testing of specific mitochondrial gene (like A1555G mtDIMA) is technically feasible ${ }^{1}$.

\section{Management}

Primary prevention of deafness will involve vaccination for rubella and mumps, proper delivery of antenatal/perinatal care and genetic counselling.

Surgical management may include insertion of grommets (ventilation tubes) in cases of persistent glue ear and myringoplasty / ossiculoplasty in those with middle ear damage caused by chronic otitis media. Antibiotics will not improve outcome of persistent glue ear. ${ }^{5}$.

Psychological management and counselling should be directed towards parents and child early in management to ensure compliance and constructive involvement.

\section{Amplification}

Prescription of hearing aids to children with impaired hearing is a complex and time consuming process and should only be undertaken by specially trained personnel. The prescription is done considering factors like amplification (power) required, frequency response, output limiting/compression and acoustic feed back suppression. Relative merits of the type of hearing aid (body worn/post aural/canal aids) should also be considered. For e.g. body worn aids are less expensive; batteries are easily available and cheap, although bulky and very visible. On the other hand, postaural and canal aids and their batteries are relatively expensive though their microphones are situated at ear level and pleasing in appearance. In the United Kingdom (UK), most children get binaural postaural aids ${ }^{2}$. Whatever the type of hearing aid prescribed, the ear mould should be custom made for the particular child. Consideration should be given for the small but growing ear canals and auricles of children during mould making.

It is very important to obtain the aided thresholds when the hearing aid fitting is complete. This will ensure that the desired amplification is actually achieved by the fitted hearing aid. In older children ( $>5$ years), it is possible to obtain insertion gain estimations ${ }^{2}$. Subsequent to hearing aid fitting, there should be an adequate period of suitable auditory training. Parents need to know that the child will take several months to get used to the hearing aid and then to recognize sounds. Thereafter it may take a year or more to produce words.

Commonly, hearing aid failures are due to inadequate amplification, too much amplification, acoustic feed back, poor compliance and lack of auditory training. In a small scale study at the Lady Ridgeway hospital, Colombo, it was found that the majority of children, who were fitted with hearing aids, do not receive any form of auditory training ${ }^{6}$. 
Hearing impaired children will also benefit from radio system communication in the classroom, electromagnetic loops in school and at home and specially designed telephone receivers compatible with hearing aids. Bone anchored hearing aids (BAHA) are now used in children with severe conductive hearing loss (e.g. those with absent ear canals with microtia and ossicular chain damage caused by chronic otitis media). In this situation, hearing aid directly conducts sounds into skull bone via an osseo-integrated metal implant.

\section{Education of the hearing impaired}

Education of the hearing impaired is an essential part of management, which should be given special attention. Teachers for the deaf and the medical team should work together to achieve the best rehabilitation. It is important to decide whether the child can attend mainstream school or he/she should be referred to a special school for the deaf. Sometimes it is necessary to refer child for sign language communication.

\section{Cochlear implants}

Cochlear implant is a device which converts sound energy into electrical impulses which are then delivered to the cochlear nerve endings inside the cochlea through an implanted multi-channel electrode. At present, it is best to consider cochlear implants only when the aided thresholds, with best possible hearing aid and auditory training, fall outside the speech range intensity and frequency. About half the children who are referred for cochlear implantation in UK do not need an implant, as they can be managed with properly fitted conventional hearing aids and auditory training ${ }^{2}$. After implantation, the device needs fine-tuning (programming) and patients need to undergo intensive auditory and speech training for many years. The following are some misconceptions about cochlear implants:

- Cochlear implants can be used instead of hearing aids. Presently, implants are only considered in patients not achieving desired results with best available conventional aids.

- The device is not visible as implant is completely inside head. This is not true as the receiving and processing parts are worn outside the body ${ }^{7}$.

- Hearing will be normal soon after implantation. This is incorrect as it takes several years of intense auditory and speech training to achieve good results $^{7}$.

\section{Conclusion}

Diagnosis and rehabilitation of the hearing-impaired child is a complex and time consuming process and should be handled by specially trained personnel. A substantial number of children can achieve adequate level of hearing and speech with properly selected conventional hearing aids, together with speech and auditory training. Those who do not benefit by this approach should be considered for cochlear implantation. All deaf children should undergo evaluation for possible aetiology and other defects and/or disabilities such as visual defects and developmental delay. Selected cases need genetic diagnosis and counselling. The multidisciplinary approach is preferred to achieve all these aims.

\section{References}

1. Bitner-Glindzicz M. Hereditary deafness and phenotyping in humans. Br Med Bull 2002; 63: 7394.

2. Snashall S. Childhood Deafness. In: Ludman H, Weight T, editors. Diseases of the Ear. 6th ed. London: Arnold, 1998; 164-81.

3. Heisent M, Meehan T, Stephens S D G. Journal of Audiological Medicine 2002; 11(2): 86-97.

4. Brookhouser P E. Sensorineural Hearing loss in Children. Paediatric Clinics of North America 1996; 43(6): 1195-216.

5. Hendley J O. Otitis Media. N Engl J Med 2002; 347:1169-74.

6. Yasawardene A D K S N, Rubasinghe M S. Outcome of usage of Hearing Aids in-group of children -A preliminary study. Abstracts of Annual Academic Sessions of Asso. Otolaryngologists of Sri Lanka 2002; 2.

7. Ballantyne J, Martin MC, Martin A. editors. The Management of the Child with hearing loss. In: Deafness. 5th ed. London 
